
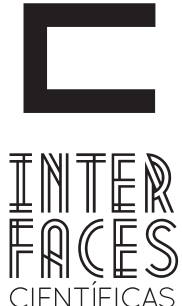

HUMANASE SOCIAIS

\title{
VALORES INDIVIDUAIS: UMA PESQUISA COM O CORPO DISCENTE DA ÁREA DE ENGENHARIA
}

Neusa Maria Bastos F. Santos Igor Polezi Munhoz"
José Wilson Franca Lelis"II

\section{RESUMO}

Os valores pessoais são capazes de influenciar ações, escolhas e comportamentos humanos, interferindo no modo que o indivíduo julga a si mesmo e aos outros (GASTALDELLO, 1999). Também afetam as decisões de carreira dos atuais e futuros profissionais que buscam inserção no mercado de trabalho. Esta pesquisa tem como principal objetivo realizar um estudo exploratório com um grupo de estudantes da área de Engenharia, pertencentes na sua maioria à geração $\mathrm{Y}$, visando: 1) identificar as diferenças e semelhanças que podem ser encontradas em relação aos seus valores pessoais; 2 ) verificar se estatisticamente há associação entre os dois diferentes tipos de valores pessoais (instrumentais e terminais) e o perfil deste alunado, considerando o gênero, idade e a formação educacional básica. 0 principal referencial teórico foi suportado pela abordagem de Valores de Rokeach (1981). O levantamento bibliográfico buscou referenciar dissertações, artigos, periódicos e textos integrais em diversas fontes com acesso a bancos de dados nacionais e internacionais. Foram sujeitos do estudo 252 alunos do curso de Engenharia de Gestão da Universidade Federal do $A B C$ (UFABC), cuja composição da amostra utilizou o critério de acessibilidade (VERGARA, 2007). A pesquisa de campo foi realizada com a aplicação do inventário de Valores de Rokeach (1981). O questionário foi construído em três partes: apresentação, perfil do participante e escala de valores terminais e instrumentais. 0 tratamento dos dados foi feito com o auxílio do pacote estatístico SPSS versão 13.0, e os resultados organizados em análise descritiva e análise inferencial. Entre os resultados obtidos, seguindo a ordem de importância destaca-se que o valor terminal primário predominante é felicidade (18\% dos entrevistados) seguido de vida confortável (17\%) e segurança familiar (10\%). Observa-se que reconhecimento social foi o valor terminal de menos incidência na opinião dos estudantes (1\%). Em relação ao valor instrumental primário, por ordem de importância destacam-se honestidade (18\%), am- 
bição (12\%) e responsabilidade (11\%). Os dois valores instrumentais menos priorizados foram obediencia (3\%) e polidez (1\%). Os testes estatísticos para um nível de significância de $5 \%$ determinaram que não há associação direta entre os diferentes valores terminais e valores instrumentais e outras variáveis de referência como gênero, idade e formação educacional. A necessidade de ampliar o espaço amostral desta investigação e investir em futuras pesquisas visando estudar a influência dos valores pessoais na inclinação profissional são subsídios importantes aqui recomendados para um melhor ajuste indivíduo - organização.

\section{PALAVRAS-CHAVE}

Valores Pessoais. Geração Y. Estudantes de Engenharia. Carreira.

\section{ABSTRACT}

Personal values can influence actions, choices and human behaviors, interfering in the way that the individual judges yourself and others (GASTALDELLO, 1999). Also, affect the career decisions of current and future professionals seeking entry into the labor market. This research has as main objective to perform an exploratory study with a group of engineering students, mostly belonging to $\mathrm{Y}$ Generation, in order to: 1) Identify the differences and similarities that can be found in relation to their personal values; 2) Verify that statistically there is an association between the two different types of personal values (instrumental and terminal) and the profile of this student body, considering the gender, age and educational base. The main theoretical approach was supported by the values of Rokeach (1981). The questionnaire was constructed in three parts: presentation, participant profile and scale of terminal and instrumental values. The data processing was done with the aid of the statistical package SPSS version 13.0, and the results organized into descriptive analysis and inferential analysis. Among the results, the order of importance is emphasized that the primary terminal value happiness is predominant ( $18 \%$ of respondents) followed by comfortable life $(17 \%)$ and family safety $(10 \%)$. It is observed that social recognition was the least incidence of terminal value in the opinion of students (1\%). Regarding the primary instrumental value, in order of importance stand out honesty (18\%), ambition (12\%) and responsability $(11 \%)$. The two instrumental values were less prioritized obedience (3\%) and politeness (1\%). Statistical tests for significance level of $5 \%$ determined that there is no direct association between different terminal values and instrumental values and other reference variables such as gender, age and education. The need to expand the sample space of this research and invest in future research to study the influence of personal values in professional inclination subsidies are important, here recommended for a better fit individual-organization.

\section{KEYWORDS}

Personal Values. Engineering Students. Y Generation. Career. 


\section{RESUMEN}

Los valores personales son capaces de influenciar acciones, escojas y comportamientos humanos, interfiriendo en el modo como el individuo juzga a si mismo y a los otros (GASTALDELLO, 1999). También afectan las decisiones de carrera de los actuales y futuros profesionales que buscan inserción en el mercado de trabajo. Esta investigación tiene como principal objetivo realizar un estudio exploratorio con un grupo de estudiantes del área de Ingeniería, pertenecientes en su mayoría a la generación Y, visando: 1) identificar las diferencias y semejanzas que pueden ser encontradas en relación a sus valores personales; 2) verificar se estadísticamente hay asociación entre los dos diferentes tipos de valores personales (instrumentales y terminales) y el perfil de este alumnado, considerando el género, edad y la formación educacional básica. El principal referencial teórico fue soportado por el abordaje de Valores de Rokeach (1981). El levantamiento bibliográfico buscó referenciar disertaciones, artículos, revistas y textos integrales en diversas fuentes con acceso a bancos de datos nacionales e internacionales. Fueron sujetos del estudio 252 alumnos del curso de Ingeniería de Gestión de la Universidad Federal del $A B C$ (UFABC), cuya composición de la amuestra utilizó el criterio de accesibilidad (VERGARA, 2007). La pesquisa de campo fue realizada con la aplicación del inventario de Valores de Rokeach (1981). El cuestionario fue construido en tres partes: presentación, perfil del participante y escala de valores terminales e in-

\section{INTRODUÇ̃̃O}

Os valores pessoais são capazes de influenciar as ações, escolhas e comportamento humano, interferindo na maneira pela qual o individuo julga a si mesmo e aos outros (GASTALDELLO, 1999). Dessa forma, eles podem ser percebidos tanto como produto como strumentales. El tratamiento dos datos fue hecho con el auxilio del paquete estadístico SPSS versión 13.0, y los resultados organizados en análisis descriptivo y análisis inferencial. Entre los resultados obtenidos, siguiendo el orden de importancia se destaca que el valor terminal primario predominante es felicidad (18\% de los encuestados) seguido de vida cómoda (17\%) y seguridad familiar (10\%). Se observa que reconocimiento social fue el valor terminal de menos incidencia en la opinión de los estudiantes (1\%). En relación al valor instrumental primario, por orden de importancia se destacan honestidad (18\%), ambición (12\%) y responsabilidad (11\%). Los dos valores instrumentales menos priorizados fueron obediencia (3\%) y cortesía (1\%). Los testes estadísticos para un nivel de significancia de un $5 \%$ determinaron que no hay asociación directa entre los diferentes valores terminales y valores instrumentales y otras variables de referencia como género, edad y formación educacional. La necesidad de ampliar el espacio de muestra de esta investigación e invertir en futuras pesquisas visando estudiar la influencia de los valores personales en la inclinación profesional son subsidios importantes aquí recomendados para un mejor ajuste individuo - organización.

\section{PALABRAS CLAVE}

Valores Personales. Generación Y. Estudiantes de Ingeniería. Carrera. ponto de referência do processo de reflexão e avaliação dos desejos (SCHWARTZ; LACEY, 1995).

Os valores pessoais afetam as reações das pessoas sobre seu trabalho (Stackman, Pinder e Connor, 2004) 
e aliados às motivações e ao talento estimulam as decisões de carreira (SCHEIN, 1993). Diante disto, as ambições da vida profissional e a satisfação das necessidades prioritárias do individuo também são impulsionadas pelos valores pessoais.

As predisposições profissionais, por sua vez, também recebem influência da centralidade do sistema cognitivo, isto é, dos valores (Gouveia et al 2001), que segundo a pluralidade científica de Milton Rokeach, são concebidos como critérios utilizados pelas pessoas para avaliar ações, indivíduos e eventos (SCHWARTZ, 2006).

Peters (2004) diz que uma revolução no local de trabalho está a caminho e seria o início da Responsabilidade Individual Renovada, isto significa que atualmente o profissional tem a oportunidade de assumir o controle de sua própria vida, tendo a escolha de atualizar- se, fazer networking e aprender onde e quando quiser. (CARNEIRO, 2010).

A busca pelo conhecimento não foi privilégio da geração atual, porém, sua propagação como ferramenta de liderança e inovação foi acentuada a partir do final da década de 1980, com o fenômeno da globalização, que impôs transformações nas estruturas organizacionais das empresas, aumentando-se a flexibilidade e aproximando-se dos anseios do consumidor.

Nesse contexto, destaca-se a importância da chamada Geração Y, formada por jovens entre 18 e 30 anos que entraram no mercado de trabalho nesta década, que apresenta maior potencial em relação às outras gerações, porque esses jovens chegam às organizações munidos de informações sobre o que acontece ao redor e estão sempre dispostos a apresentar soluções criativas. Esta geração possui características específicas: predominantemente virtuais; com comportamen- to empreendedor; inovadoras; de liderança; criativas; responsáveis; com paixão na área atuante; com visão de futuro; de persistência; de coragem para assumir riscos; de facilidade de expressão (CARNEIRO, 2010).

O ajuste das exigências trabalhistas à realidade do mercado faz com que os antes determinados vínculos empregatícios se transformem em parcerias, contribuindo para o surgimento de formas flexíveis de trabalho. Hoje, os cargos deixaram de ser a referência para a gestão de pessoas, dando lugar à competência independente da sua posição na empresa.

Dessa forma, foi feita a análise dos valores pessoais dos estudantes da área de Engenharia, considerando os fatores pessoais que influenciam na determinação dos deles.

A amostra pode ser considerada heterogênea em relação as suas características socioculturais e econômicas, embora ela faça parte de um grupo que frequenta a mesma instituição de ensino superior, com aspirações e desejos semelhantes, existem diversidades no poder aquisitivo, que embora não sejam identificadas na pesquisa, podem ser observadas na política de cotas adotada pela instituição, além das diversidades com relação a crenças e costumes também observadas e a variações das ênfases do curso de Engenharia de Gestão, mas essas variações em si não interferem no objetivo maior desse trabalho.

Este estudo é comumente realizado na área de Administração, no entanto existem poucas pesquisas feitas na área de Engenharia. Visando obter uma resposta exploratória, investigar-se-á um grupo particular de indivíduos, alunos de Engenharia da Universidade Federal do ABC, para determinar seus valores pessoais e quais são seus fatores determinantes.

\subsection{FORMULACÃO DO PROBLEMA}

A meta proposta pela pesquisa traz dois questionamentos a serem considerados: "Quais as diferenças e semelhanças que podem ser encontradas no grupo de

alunos da área de engenharia em relação aos seus valores instrumentais e terminais? E quais os fatores individuais que influenciam na determinação dos valores pessoais?”. 


\subsection{OBJETIVOS}

O objetivo da pesquisa é realizar um estudo de campo com universitários da Universidade Federal do $A B C$, para determinar seus valores pessoais, obtidos através da aplicação de questionários em alunos do

\subsection{IMPORTÂNCIA E CONTRIBUIÇÃO}

Existe a necessidade de estudos e pesquisas na área de valores pessoais, pois estes afetam as decisões de carreira.

A pesquisa busca compreender os valores considerados mais importantes para os jovens de hoje, pertencentes à chamada geração $\mathrm{Y}$, devido as suas características específicas, e se esses valo- curso de Engenharia, na sua maioria pertencentes à geração $\mathrm{Y}$, e relacioná-los aos aspectos individuais e preferências da amostra.

res são os mesmos esperados pelas organizações que futuramente empregarão esses indivíduos, pois eles serão cobrados pelo mercado de trabalho a terem uma visão crítica e devem ter na bagagem soluções criativas, inovadoras e ousadas que possam impactar nas decisões da empresa, sendo fundamental estudar a maneira como essas relações tem se estabelecido.

\section{FUNDAMENTAÇ̃̃O TEÓRICA}

Os valores não são apenas palavras, eles guiam e orientam nosso comportamento e afetam nossas experiências cotidianas, portanto, é fundamental o alicerce em um sistema conceitual competente, composto por conceitos de valores, apresentados à luz das contribui-

\section{A. ESTUDO DOS VALORES HUMANOS À LUZ DAS CONTRIBUICÕES DE ROKEACH}

Ao definir o termo valor deve-se restringir seu significado à realidade estudada, pois ele carrega consigo uma dimensão pluri-significados, vinculada a diferentes situações do cotidiano, expressando desde um critério essencial para o individuo escolher o que considera mais importante, ou que não pode ser dispensado, um padrão fundamental de comportamento até objetos de valor (apego sentimental, estima ou preço do bem em termos monetários) (SCHWARTZ; LACEY, 1995). ções de Milton Rokeach, professor da Universidade de Minnesota e considerado um dos maiores especialistas mundiais no estudo de valores, e sua respectiva taxionomia em valores instrumentais e terminais, necessários à compreensão do objeto de estudo desta pesquisa.

Poucos trabalhos apresentam um conceito que atenda as expectativas do objeto de estudo e que se afaste do senso comum. Algumas das polêmicas envolvidas residem na questão relacionada ao juízo de valor, inconcebível em pesquisas científicas, pois a busca deve ser guiada pela compreensão e análise dos fenômenos e não sua apreciação e avaliação entre certo e errado (QUIVY; CAMPENHOUDT, 1998). É necessário esclarecer que o objetivo desta pesquisa não está relacionado com juízo de valores. 
Alguns estudiosos de valores apresentaram suas definições, como Kluckohn, Rokeach e Schwartz, sendo que delimitam suas pesquisas em valores humanos. Kluckohn (1951, p. 443), parte da interação social e define valor como "uma concepção explícita, ou implícita, distinta, ou característica de um grupo do que é desejável, que influencia a seleção de modos, meios e finalidades da ação acessível".

Conforme a definição de Kluckohn (1951), um valor supõe um código ou uma norma que tem alguma persistência ao longo do tempo, com seu uso. Esse valor é aceito pelo grupo, de forma que o desejável é contrastado com o desejado.

Schwartz (2005) assevera que valores são aquilo que os indivíduos tomam como importante em suas vidas. Afirma que valores são crenças; um construto motivacional; que transcendem situações e ações específicas; que guiam a seleção e avaliação de ações, políticas, pessoas e eventos; e que os valores são ordenados pela sua importância relativa perante os demais.

Ainda segundo Schwartz, os valores formam um conjunto de metas conscientes dos indivíduos ou sociedades que devem atender aos três requisitos universais: as necessidades biológicas dos indivíduos, os requisitos de interação social e os requisitos para o correto funcionamento e sobrevivência dos grupos (SCHWARTZ, 2001).

Rokeach apresenta sua definição de "valor" e "sistema de valor" como sendo:

\section{B. VALORES TERMINAIS E VALORES INSTRUMENTAIS}

Rokeach (1981) apresenta uma distinção entre dois tipos de valores, os valores instrumentais dos valores terminais. Os valores terminais, também chamados de valores finais, são os estados finais preferidos de ser e estar, enquanto os valores instrumentais correspon-
Um valor é uma crença duradoura de que um modo específico de conduta ou estado final de existência é pessoal ou socialmente preferível a um modo de conduta ou estado final de existência oposto ou contrário. Um sistema de valores é uma duradoura organização de crenças referentes a modos preferenciais de conduta ou estados de existência considerados com base em uma escala de importância relativa (ROKEACH, 1973, p. 5).

Dentro dessa concepção, Hofstede (1997) afirma que os seres humanos compartilham valores semelhantes, independentemente da cultura nacional, em conformidade ao preconizado por Rokeach (1981), porém o que muda são suas práticas. Rokeach afirma que os fatores sociais (cultura, classe socioeconômica, educação, ocupação e opção religiosa e política) restringem as variações nos padrões de valores das pessoas, enquanto os fatores pessoais, como a personalidade, aumentam as variações.

Nessa abordagem, o valor é concebido como um guia transcendente que se aplica a qualquer objeto ou situação a qual o individuo é submetido, já que desde que o valor é internalizado, seja de forma consciente ou inconsciente, torna-se um critério ou modelo para conduzir a ação; justificar ações e atitudes de si e dos outros; julgar; e comparar-se aos demais (GASTALDELLO, 1999).

Desta forma, Rokeach (1981) afirma que os indivíduos diferem nas práticas dos valores devido aos graus de importância atribuídos a cada um deles, ordenando-os e articulando-os entre si dentro de um sistema de valores, que é acionado diante das escolhas pessoais. Quando isso ocorre, os valores considerados mais relevantes no sistema predominam sobre os demais.

dem aos modos preferidos de conduta social ou comportamentos para o alcance dos objetivos pessoais.

Um valor terminal pode ser identificado pela seguinte sentença: "eu creio que tal e tal estado de exis- 
tência (por exemplo, a salvação, um mundo de paz) é pessoal e socialmente um valor que se deve lutar para obter". Um valor instrumental, por sua vez, pode ser identificado pela afirmativa: "eu creio que tal e tal modo de conduta (a honestidade, a coragem, por exemplo) é pessoal e socialmente preferível em todas as situações com respeito a todos os objetos" (ROKE$\mathrm{ACH}, 1966)$. Dessa maneira, apenas os valores que podem ser inseridos em cada uma das frases podem ser classificados como terminais ou instrumentais (ABRAHIM, 2008).

Segundo Rokeach (1973), os valores finais que as pessoas geralmente costumam defender não passam de doze, enquanto o número de valores instrumentais é maior, mas não chega a cem.

Dolan e Garcia (2006) propõem a subdivisão de dois grupos. Nessa proposta, os valores instrumentais são compostos por valores ético-morais e valores de competição enquanto que os valores finais são divididos entre valores pessoais e valores ético-sociais.

Os valores ético-morais se referem a formas de conduta que são necessárias para que alcancemos nossos valores finais. Eles possuem duas características peculiares: são colocados em ação na relação com outras pessoas e em pessoas emocionalmente equilibradas quando esses valores são defendidos, mas não se traduzem num comportamento conseqüente ou coerente, eles tendem a gerar sentimentos de culpa e vergonha. Como exemplo tem-se a honestidade, sinceridade, lealdade, solidariedade e outros.

Valores de competição são aqueles valores necessários para competir, geralmente mais in- dividuais, embora também sejam condicionados socialmente. Não estão relacionados diretamente com moralidade ou culpa. Entre os valores de competição destaca-se a iniciativa, a lógica e a imaginação.

Valores pessoais são aqueles que o indivíduo almeja para si mesmo, como felicidade, sucesso pessoal e reconhecimento. Valores ético-sociais são aqueles que constituem aspirações ou propósitos que beneficiam a sociedade como um todo, entre os quais, justiça social e respeito ao meio ambiente.

A distinção entre valores instrumentais e finais é útil para a mensuração em duas escalas, com 18 valores em cada, já que sem a divisão haveria uma lista única com 36 valores, o que dificultaria a classificação em ranking, considerando a dificuldade na ordenação nos mesmos. Rokeach (1981) realizou pesquisas com grupos de controle para chegar a essa divisão, após realizar testes e chegar ao melhor resultado de confiabilidade de instrumento. Embora exista a separação em duas listas, não se desconsidera a inter-relação entre os valores presentes. Nas Figuras 1 e 2 são apresentados os valores que compõem as referidas listas.

Diante do exposto, é possível afirmar que os valores representam a centralidade do sistema cognitivo (GOUVEIA et al, 2001), representando abstrações que estimulam atitudes e refletindo-se nos comportamentos, logo, os valores humanos são capazes de influenciar as ações, escolhas e moldar o comportamento humano, tanto no que diz respeito a estruturas mais gerais, como a vida quanto em contextos mais específicos, como o trabalho. 
Figura 1. Valores instrumentais e seus respectivos significados

\begin{tabular}{|c|c|}
\hline Valores instrumentais & Significado \\
\hline Modos preferíveis de conduta & Como prefere se comportar para consecução de seus objetivos \\
\hline Ambicioso & Trabalhador incansável, com aspirações \\
\hline Liberal & Mentalidade aberta, aceitar opiniões e atitudes diferentes da sua, ser flexível \\
\hline Capaz & Competente, eficiente \\
\hline Animado & Alegre, jovial \\
\hline Asseado & Limpo, arrumado, bem apresentável \\
\hline Corajoso & Pronto a defender seus valores, suas ideias, suas vontade \\
\hline Tolerante & Disposto a perdoar outros, a aceitar as coisas como elas são \\
\hline Prestativo & Trabalhando para o bem estar de outrem \\
\hline Honesto & Sincero, verdadeiro \\
\hline Imaginativo & Ousado, criativo \\
\hline Independente & Autoconfiante, autossuficiente, poder fazer as coisas por si \\
\hline Intelectual & Inteligente, pensativo, estudioso \\
\hline Lógico & Consciente, racional, que tem raciocínio rápido \\
\hline Amoroso & Afetuoso, carinhoso \\
\hline Obediente & Submisso, sabe obedecer as ordens dos superiores, respeitoso \\
\hline Polido & Cortês, educado \\
\hline Responsável & Fidedigno, de confiança, assume suas obrigações \\
\hline Autocontrolado & Contido, com autodomínio, prudente, ponderado \\
\hline
\end{tabular}


Figura 2. Valores terminais e seus respectivos significados

\begin{tabular}{|c|c|}
\hline Valores terminais & Significado \\
\hline Estados preferidos de ser/estar & Os objetivos de existência \\
\hline Uma vida confortável & Uma vida próspera, dinheiro, bens, conforto material \\
\hline Uma vida excitante & $\begin{array}{l}\text { Uma vida estimulante, ativa, não-monótona, não-rotineira, com desafios a se- } \\
\text { rem vencidos }\end{array}$ \\
\hline Um sentimento de realização & $\begin{array}{l}\text { Contribuição duradoura, auto-realização, estar satisfeito consigo, gostar da vida } \\
\text { que leva }\end{array}$ \\
\hline Um mundo de paz & Livre de guerras e conflitos, vida harmoniosa com a família, vizinhos, no trabalho \\
\hline Um mundo de beleza & Beleza da natureza e da arte \\
\hline Igualdade & Fraternidade, oportunidades iguais, direitos iguais \\
\hline Segurança familiar & Cuidando dos que the são caros, da família e pessoas queridas \\
\hline Liberdade & Independência, livre escolha, poder fazer as coisas como você quer e gosta \\
\hline Felicidade & Contentamento, satisfação, alegria, estar de bem com a vida \\
\hline Harmonia interior & Livre de conflitos interiores \\
\hline Amor maduro & Intimidade sexual e espiritual \\
\hline Segurança nacional & Proteção contra ataques, segurança do país \\
\hline Prazer & Uma vida agradável, calma, satisfação pessoal \\
\hline Salvação & Uma vida eterna, salva, espiritualidade, ter fé em Deus \\
\hline Autorrespeito & Autoestima, gostar de si mesmo \\
\hline Reconhecimento social & Ter o respeito das demais pessoas, ser admirado pelos outros \\
\hline Amizade verdadeira & $\begin{array}{l}\text { Camaradagem intima, fazer amigos verdadeiros, com quem possa contar e que } \\
\text { possam contar com você }\end{array}$ \\
\hline Sabedoria & Uma compreensão adulta da vida \\
\hline Autocontrolado & Contido, com autodomínio, prudente, ponderado \\
\hline
\end{tabular}




\section{GERAÇÃO Y}

Quando se fala em geração Y, deve-se em primeiro lugar entender o contexto histórico em que a ela se situa, bem como entender o seu perfil. De acordo com Kupperschmidt (2000), as diferenças entre gerações em uma empresa, por exemplo, se não compreendidas, podem gerar tensão, confronto, insatisfação e diminuição na produtividade.

A geração $Y$ vem sendo foco de muitos estudos nos últimos anos devido o fato de ser a mais recente geração a entrar no mercado de trabalho e, entender como atraí-la e retê-la em uma empresa, torna-se um desafio. A seguir será abordado o conceito de geração como um todo, mas focando-se especialmente na geração Y.

Geração refere-se ao conjunto de indivíduos nascidos em uma mesma época, sendo que cada geração possui determinadas características, valores e princípios distintos umas das outras. Começando na in- fância, as gerações formam como se fosse um ciclo e sempre devem adaptar-se para que possam conviver umas com as outras de forma harmoniosa (CARNEIRO, 2010).

Segundo Strauss e Howe (1992) uma geração é influenciada pelos eventos e circunstâncias as quais seus membros experimentaram e vivenciaram em certas fases da vida. 0 material pesquisado nomeia seis gerações principais: Seniors, Builder, Baby Boomers, Geração X, Geração Y e Geração Z. Na Figura 3 apresentam-se as atuais gerações juntamente com o ano de nascimento e idade.

Os membros pertencentes à geração $Y$ são indivíduos que nasceram entre os anos de 1982 e 2000 (RUGIMBANA, 2007). A Geração Y faz parte do chamado ciclo idealista, porque pode-se fazer uma correlação entre as suas características principais, o empreendedorismo, a independência e a honestidade. Alguns

Figura 3. Atuais gerações

\begin{tabular}{|l|l|l|l|}
\hline \multicolumn{1}{|c|}{ Descrição } & \multicolumn{1}{c|}{ Nascimento } & Idade & \multicolumn{1}{c|}{ Alguns exemplos de personalidades } \\
\hline Seniors & Antes de 1925 & $84+$ & Mahatma Gandhi (1869 - 1948); Albert Einstein (1879-1955) \\
\hline Builders & $1926-1945$ & $83-64$ & $\begin{array}{l}\text { Mikhail Sergeyevich Gorbachev (1931); Ernesto Guevara de la Serna, } \\
\text { mais conhecido por Che Guevara ou El Che (1928 -1967) }\end{array}$ \\
\hline Baby Boomers & $1946-1964$ & $63-45$ & Ayrton Senna da Silva; Barack Hussein Obama II (1961) \\
\hline Geração X & $1965-1981$ & $44-28$ & Leonardo Wilhelm DiCaprio; Kimi Raikkonen, 29 anos \\
\hline Geração Y & $1982-2000$ & $27-9$ & $\begin{array}{l}\text { Daniel Radcliffe - ator jovem mais rico do mundo; Beyoncé Knowles, } \\
\text { 27 anos }\end{array}$ \\
\hline Geração Z & $2001+$ & $8-$ & $\begin{array}{l}\text { Mercy James (5 anos) - filha da Madonna; Benicio Huck - filho da } \\
\text { Angélica e Luciano Huck }\end{array}$ \\
\hline
\end{tabular}

Fonte: Adaptado de Carneiro (2010) do original de McCrindle (2002) 
autores, como McCrindle (2002) e Fields (2008), afirmam que a condição social, política e econômica é um fator diferencial no comportamento das gerações. Cada geração possui diferentes características pessoais e estilo de vida em relação aos valores, família, educação, comunicação e finanças.

No ambiente de trabalho, são vistos como funcionários flexíveis, que gostam de trabalhar em equipe e dos desafios que novas tarefas e oportunidades podem trazer. Em relação à sua interação no ambiente de trabalho, é predominante o uso do ambiente virtual. Juntando sua forma de interação e suas características, esta geração traz nova atitude e com isso, pode gerar alguns conflitos devido à sua diferença comportamental, características, valores e estilo de liderança. Nota-se que algumas revistas como Você S/A, Veja, Harvard Business Review, entre outras, publicam artigos periodicamente sobre a gestão destes profissionais para tentar compreender como lidar com este comportamento e proporcionar melhores práticas de liderança.

De acordo com Martin (2005), "os Y's veem a educação como a chave para o sucesso", ou seja, consideram a universidade como um lugar de crescimento intelectual e espiritual. Como método de aprendizagem e estudo, preferem os trabalhos em grupo, onde é possível interagir, colaborar e dividir opiniões e responsabilidades (SHIH; ALLEN, 2007).

A geração Y é vista como um grupo que possui valores éticos muito fortes, bem como uma consciência social nunca antes vista em nenhuma juventude. Ética, meio ambiente, aquecimento global e pobreza são temas de grande preocupação e discussão na roda de grupos desses jovens (PARTRIDGE, 2006)
Ao juntar os problemas e expectativas da Geração Y, encontra-se uma nova visão de trabalho, uma nova forma de trabalho encontrada no mundo atual, onde interesses dos trabalhadores e empregadores tomam uma nova perspectiva. (CARNEIRO, 2010)

\begin{abstract}
Uma revolução do local de trabalho está a caminho. Nenhuma pessoa sensata pretende mais passar toda a sua vida em uma única empresa. Alguns chamam essa mudança de "fim da responsabilidade corporativa". Eu a chamo de... "O início da Responsabilidade Individual Renovada". Uma extraordinária oportunidade de assumir controle de nossas próprias vidas. (PETERS, 2004, p. 8)
\end{abstract}

O autor destaca que cada indivíduo é responsável pelo seu próprio trabalho e a importância da gestão do conhecimento, que é de grande valor para as empresas. Essas tendências geram impactos na geração de menos empregos formais e o surgimento de um novo formato de trabalho informal na sociedade atual, o sistema da empregabilidade.

\begin{abstract}
A empregabilidade é um termo recente que tem sua definição a condição de ser empregável, isto é, de dar ou conseguir emprego para seus conhecimentos, habilidades e atitudes intencionalmente desenvolvidos por meio de educação e treinamento sintonizados com as novas necessidades do mercado de trabalho. (MINARELLI, 2007, p.4)
\end{abstract}

São crescentes os incentivos aos profissionais para que se qualifiquem com maior rapidez e intensidade. As empresas esperam que esta geração seja mais informada, empreendedora e interessada no processo de aprendizagem contínua. A frente desse cenário em que o perfil do profissional é prioritariamente informacional, a geração vive certa pressão quanto ao seu futuro dentro da empresa, que depende da gestão do conhecimento. 


\section{AÇÕES METODOLÓGICAS}

0 projeto baseia-se em um estudo exploratório, integrando a pesquisa bibliográfica e a pesquisa de campo. A investigação iniciou-se com a busca de referências nas áreas de engenharia, psicologia, sociologia e administração em banco de dados nacionais e internacionais, como SCielo, Proquest, Ebsco, Lilacs e outros, assim como o acervo digital

\section{A. PLANO AMOSTRAL}

A escolha dos participantes deu-se inicialmente pelo critério de acessibilidade (Vergara, 2007) ao se optar pela investigação de universitários da Universidade Federal do $A B C$ (UFABC), instituição pública de ensino superior, pertencentes à geração $Y$.

A UFABC organiza os cursos por áreas de conhecimento. Dentre elas tem-se o Centro de Engenharia e Ciências Sociais Aplicadas com os cursos de graduação oferecidos em Engenharia, nas modalidades: Aeroespacial; Ambiental e Urbana; Bioengenharia;

\section{B. COLETA DE DADOS}

A hierarquia de valores pessoais do grupo analisado foi identificada pela utilização da Escala de Valores de Rokeach ou Rokeach Value Survey (RSV), pois se considera o instrumento mais apropriado para a mensuração de valores e pela sua parcimônia ante aos demais instrumentos como o Inventário de Valores Humanos de Schwartz, composto por 56 questões.

\section{APLICAÇÃO DO INSTRUMENTO DE PESQUISA}

O questionário foi composto por três partes. A primeira é a apresentação do estudo, seguida pelo perfil do entrevistado, formada por 10 perguntas e a terceira corresponde ao instrumento de pesquisa, a escala de valores de Rokeach. Este foi aplicado em disciplinas específicas do curso de Engenharia de Gestão, de universidades brasileiras. Após o levantamento das obras, decidiu-se pela utilização de questionário como forma de coleta de dados. Desta forma, a pesquisa de campo é feita através da aplicação do inventario de avaliação da escala de valores instrumentais e finais (ou terminais), elaborado por Rokeach (1973).

Energia; Gestão; Informação; Instrumentação, Automação e Robótica e Materiais e na área de Ciências Sociais, são oferecidos os bacharelados em Ciências Econômicas e Políticas Públicas. 0 grupo de discentes selecionados compõe-se de alunos do curso de Engenharia de Gestão. A amostra é não probabilística porque durante a coleta de dados os questionários foram distribuídos em sala de aula aos alunos presentes e dispostos a participar da pesquisa, apresentando distribuição aleatória. Dessa forma, se obteve como resultado uma amostra com 252 participantes.

O inventario RVS é composto por duas listas com 36 valores, sendo 18 terminais e 18 instrumentais, permitindo aos indivíduos que atribuam os graus de importância a cada um dos valores das duas listas em escala decrescente, de um a 18. A pontuação de menor valor corresponde à maior importância e a pontuação maior representa a menor importância.

entre dezembro de 2009 e março de 2010. A duração de aplicação do questionário em cada sala ocorreu em média de 30 minutos.

A aplicação do questionário ocorreu da seguinte maneira: 1) Foi entregue aos alunos dentro da sala 
de aula uma folha individual contendo a apresentação do estudo na capa, seguida de um questionário, necessário para identificar o perfil do participante e na seqüencia o questionário de valores, que segue o

\section{PROCEDIMENTOS DE ANÁLISE}

Após a coleta de dados os questionários foram tabulados, formando um banco de dados no Excel. 0 tratamento dos dados foi feito com o auxilio do pacote estatístico SPSS versão 13.0 e os resultados organizados em análise descritiva e análise inferencial.

Utilizando-se a análise descritiva, identificou-se o valor terminal primário (classificado como 1 - o mais importante) e o secundário (classificado como 2 - o segundo mais importante), e o valor instrumental primário e secundário de cada entrevistado. Os indivíduos que colocaram mais de um valor como o mais importante ou o segundo mais importante não foram considerados no cálculo dessas variáveis. Em seguida, realizaram-se as análises descritivas de cada uma

\section{RESULTADOS DA PESQUISA}

A amostra analisada, composta por 252 sujeitos, conforme exposto no item 3.2, integra discentes do

\section{A. PERFIL DOS PARTICIPANTES}

A Figura 4 mostra que a maioria dos entrevistados (92\%) tem entre 18 e 25 anos, pertencentes à geração Y.

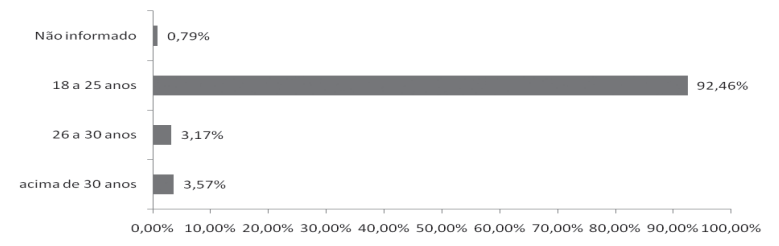

Figura 4. Análise descritiva da idade padrão da Escala de Valores de Rokeach. 2) Após o término as folhas foram recolhidas para serem analisadas. É importante ressaltar que não foram coletados dados que identificassem os participantes.

das variáveis pesquisadas (perfil e valores) de forma independente, utilizando gráficos de barras.

Para as análises inferenciais foram utilizados testes de Qui-quadrado avaliando se há associação dos valores terminais e instrumentais com o gênero, a faixa etária e a formação anterior. 0 teste de Qui-quadrado foi utilizado por ser um teste não paramétrico que permite avaliar a associação entre duas variáveis categóricas (nominais ou ordinais) através de uma tabela de contingência.

Após a obtenção dos resultados, estes foram comparados aos resultados de estudos realizados anteriormente com alunos de outros cursos em outras instituições de ensino.

curso de engenharia da Universidade Federal do $A B C(U F A B C)$.

Observa-se na Figura 5 que a maioria dos entrevistados é homens (59\%) e que uma quantidade considerável de entrevistados não informou o gênero (17\%).

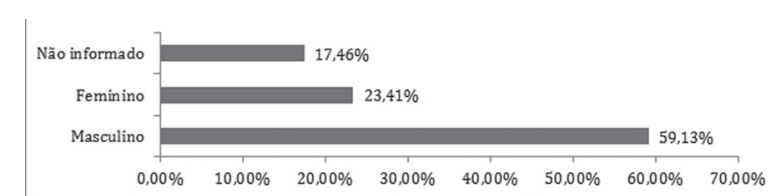

Figura 5. Análise descritiva do gênero 
A maioria dos entrevistados concluiu apenas o ensino médio (68\%), $24 \%$ concluíram o ensino técnico

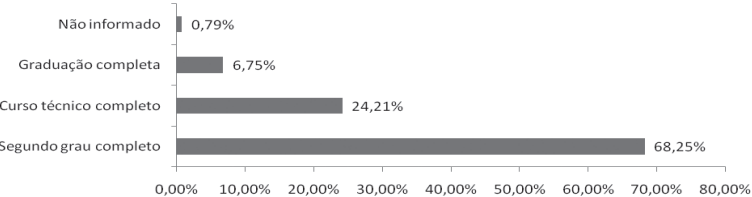

Figura 6. Análise descritiva da formação anterior.

\section{B. A HIERARQUIA DOS SISTEMAS DE VALORES}

A Figura 7 mostra que o valor terminal primário predominante é a felicidade, sendo o mais importante para 46 entrevistados (18\%). Para 17\%, a vida confor- de nível médio e $7 \%$ possuem outra graduação completa, conforme mostrado na Figura 6.

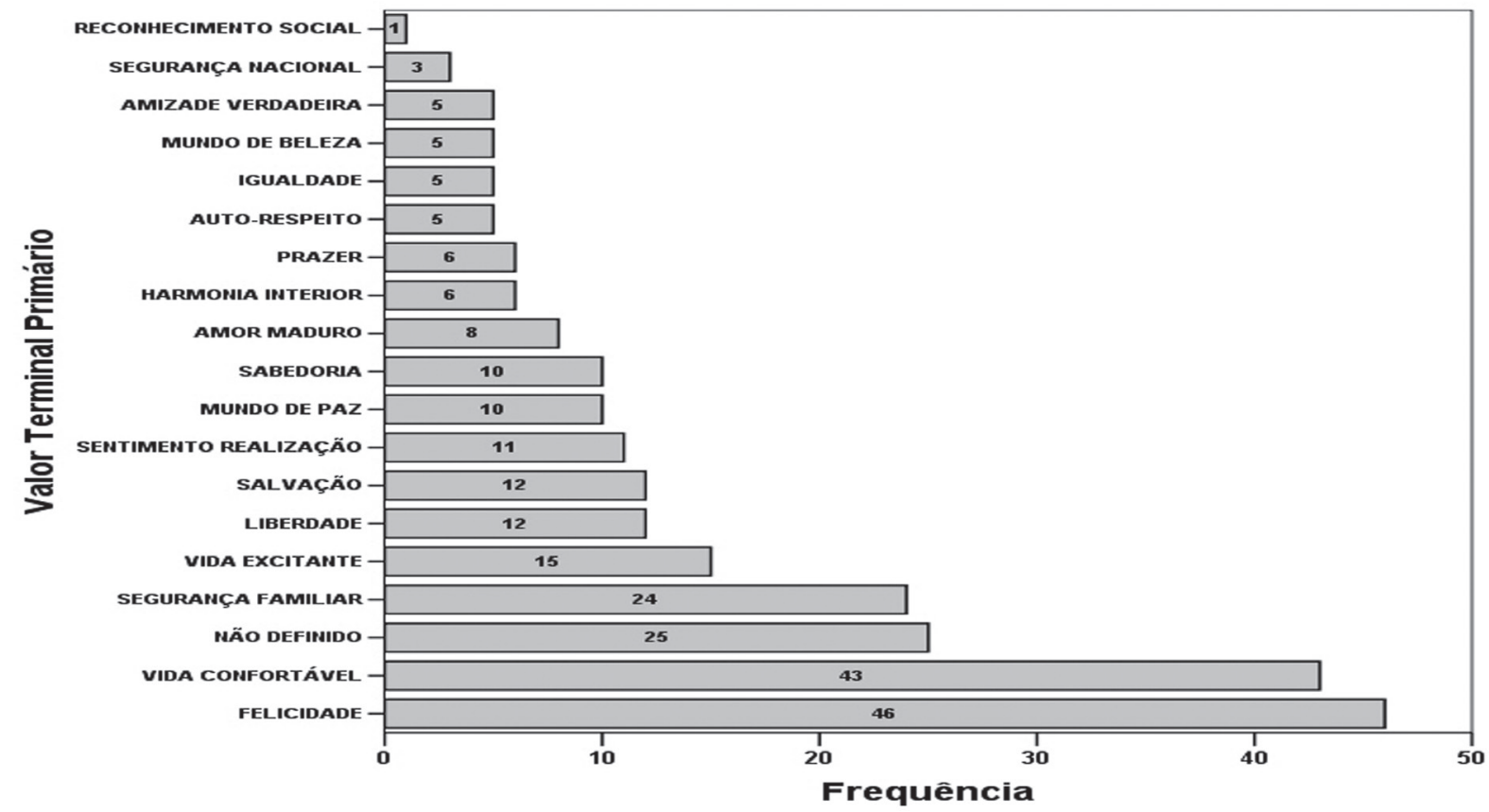

Figura 7. Análise descritiva do valor terminal primário

tável é o valor mais importante e a segurança familiar foi considerado o valor mais importante para 24 entrevistados (10\%).
Na Figura 8 pode-se observar que o valor terminal secundário predominante é a felicidade, sendo o segundo mais importante para 27 entrevistados (11\%), para $8 \%$ a vida confortável é o segundo valor mais importante, 0 sentimento de realização foi considerado o segundo valor mais importante para 18 entrevistados (7\%). 


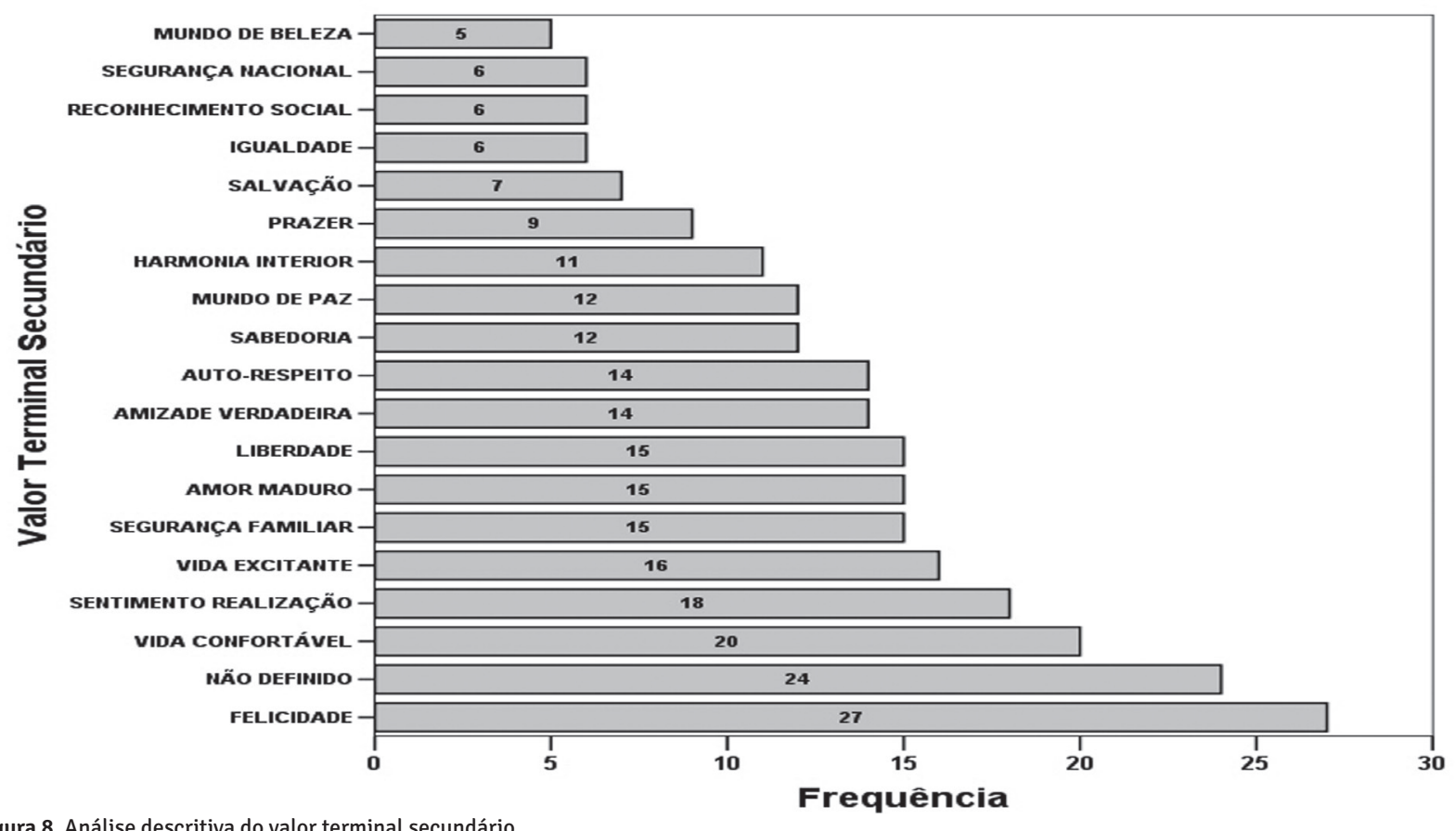

Figura 8. Análise descritiva do valor terminal secundário

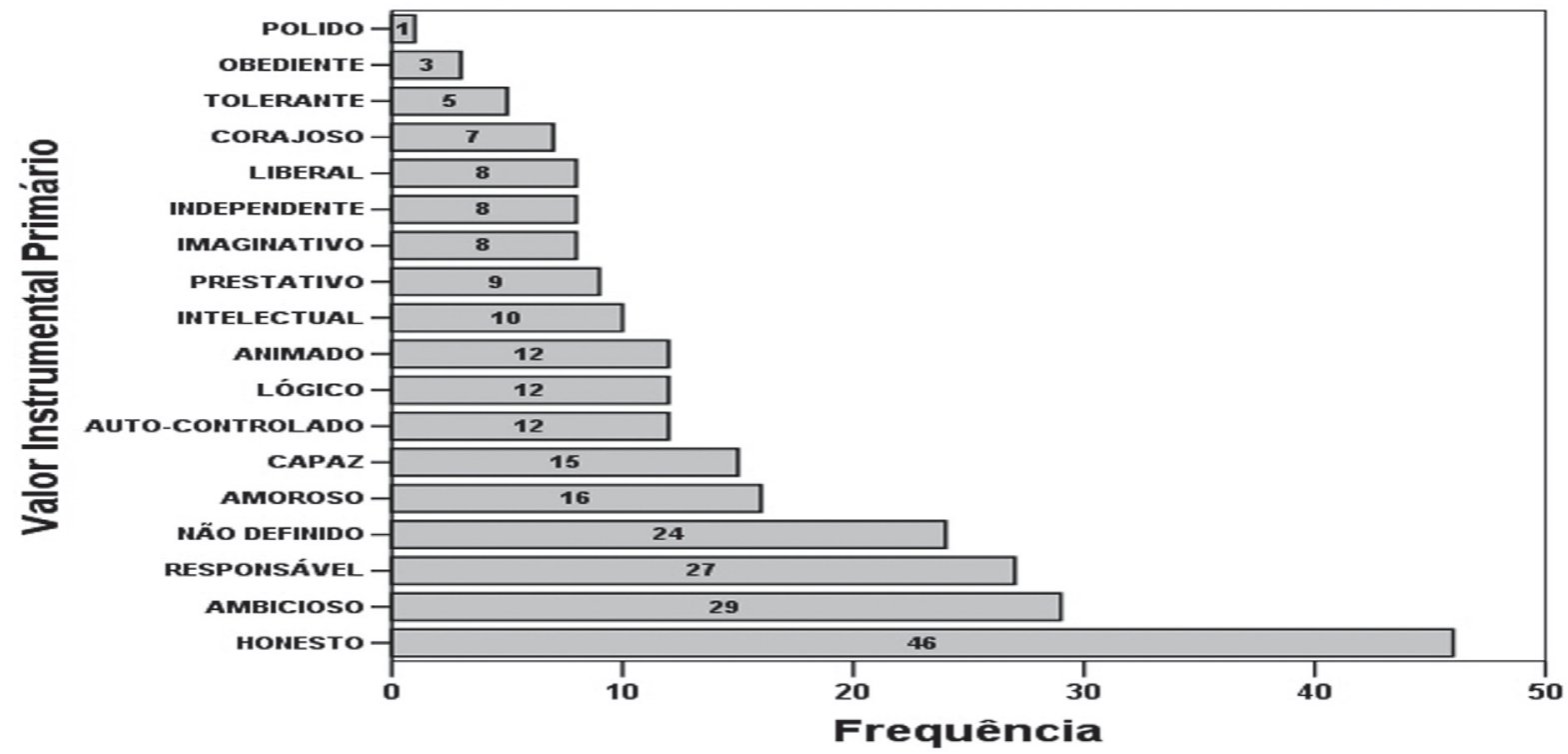

Figura 9. Análise descritiva do valor instrumental primário 
A Figura 9 mostra que o valor instrumental primário predominante é a honestidade, sendo o mais importante para 46 entrevistados (18\%). Para 12\% a ambição é o valor mais importante, a responsabilidade foi considerada o valor mais importante para 27 entrevistados (11\%).
Nota-se na Figura 10 que o valor instrumental secundário predominante é a responsabilidade, sendo o segundo mais importante para 33 entrevistados (13\%). Para $10 \%$ a honestidade é o segundo valor mais importante, a prestatividade foi considerada o segundo valor mais importante para 20 entrevistados (8\%).

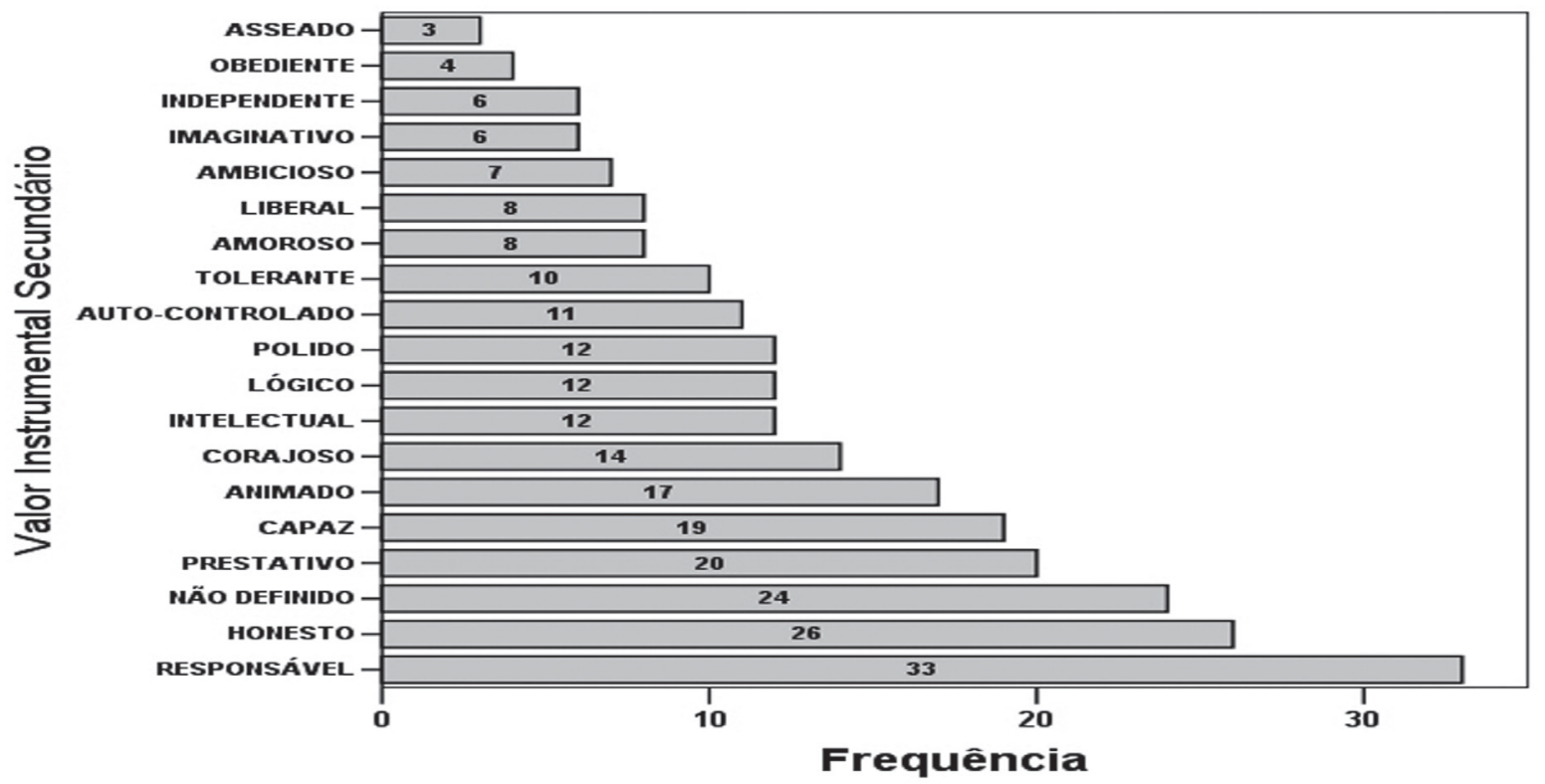

Figura 10. Análise descritiva do valor instrumental secundário

\section{INFLUÊNCIAS DO PERFIL DO PARTICIPANTE NO SISTEMA DE VALORES}

A seguir são apresentados os resultados de contingência entre os valores instrumentais e terminais e 0 perfil do participante.

Verifica-se na Tabela 1 que, ao nível de significância de 5\%, não há associação entre o valor terminal primário e a idade. A Tabela 2 apresenta a contingência entre o valor terminal primário e o gênero, e o resultado do teste de Qui-quadrado.

Através da análise da Tabela 2, considerando-se o nível de significância de $5 \%$, é possível afirmar que não há asso- ciação entre o valor terminal primário e o gênero. Na Tabela 3 tem-se a contingência entre o valor terminal primário e a formação anterior, e o resultado do teste de Qui-quadrado.

Pode-se verificar na Tabela 3 que, ao nível de significância de 5\%, não há associação entre o valor terminal primário e a formação anterior. Na Tabela 4 é apresentada a contingência entre o valor instrumental primário e a idade, e o resultado do teste de Qui-quadrado. 
Tabela 1:Análise de contingência entre o valor terminal e a idade

\begin{tabular}{|l|l|l|l|}
\hline & \multicolumn{2}{|c|}{ IDADE } & \multirow{2}{*}{ TOTAL } \\
\cline { 1 - 3 } VALOR TERMINAL & $\begin{array}{l}\text { Até 25 } \\
\text { anos }\end{array}$ & $\begin{array}{l}\text { Mais de } \\
25 \text { anos }\end{array}$ & \\
\hline Felicidade & 45 & 1 & 46 \\
\hline Segurança Familiar & 20 & 4 & 24 \\
\hline Vida Confortável & 41 & 2 & 43 \\
\hline Vida Excitante & 14 & 1 & 15 \\
\hline Outros & 93 & 5 & 98 \\
\hline TOTAL & 213 & 13 & 226 \\
\hline
\end{tabular}

Nota. Qui-quadrado=6,556; DF=4; P_valor=0,161.
Tabela 2: Análise de contingência entre o valor terminal e o gênero

\begin{tabular}{|l|l|l|l|}
\hline & \multicolumn{2}{|c|}{ GÊNERO } & \multirow{2}{*}{ TOTAL } \\
\cline { 1 - 3 } VALOR TERMINAL & Masculino & Feminino & \\
\hline Felicidade & 27 & 11 & 38 \\
\hline Segurança Familiar & 11 & 9 & 20 \\
\hline Vida Confortável & 26 & 8 & 34 \\
\hline Vida Excitante & 8 & 5 & 13 \\
\hline Outros & 63 & 21 & 84 \\
\hline TOTAL & 135 & 54 & 189 \\
\hline
\end{tabular}

Nota. Qui-quadrado=4,219; DF=4; P_valor=0,377.

Tabela 3: Análise de contingência entre o valor terminal e a formação anterior

\begin{tabular}{|l|l|l|l|l|}
\hline & \multicolumn{3}{|c|}{ FORMAÇÃO ANTERIOR } & \multirow{2}{*}{ TOTAL } \\
\cline { 1 - 4 } \multicolumn{1}{|c|}{ VALOR TERMINAL } & $2^{\circ}$ Grau Completo & Curso Técnico Completo & Graduação Completa & \\
\hline Felicidade & 32 & 11 & 3 & 46 \\
\hline Segurança Familiar & 15 & 6 & 3 & 98 \\
\hline Vida Confortável & 34 & 7 & 2 & 24 \\
\hline Vida Excitante & 10 & 3 & 2 & 43 \\
\hline Outros & 64 & 29 & 5 & 15 \\
\hline TOTAL & 155 & 56 & 15 & 226 \\
\hline
\end{tabular}

Nota. Qui-quadrado=6,170; DF=8; P_valor=0,628.

Verifica-se na Tabela 4 que, ao nível de significância de 5\%, não há associação entre o valor instrumental primário e a idade. Na Tabela 5 mostra-se a contingência entre o valor instrumental primário e o gênero, e o resultado do teste de Qui-quadrado.

Através da análise da Tabela 5, ao nível de significância de 5\%, conclui-se que não há associação entre o valor instrumental primário e o gênero. A Tabela 6 apresenta a contingência entre o valor instrumental primário e a formação anterior, e o resultado do teste de Qui-quadrado

Verifica-se na Tabela 6 que, ao nível de significância de 5\%, não há associação entre o valor instrumental primário e a formação anterior. 
Tabela 4: Análise de contingência entre o valor instrumental e a idade

\begin{tabular}{|c|c|c|c|}
\hline & \multicolumn{2}{|c|}{ GÊNERO } & \multirow[t]{2}{*}{ TOTAL } \\
\hline $\begin{array}{c}\text { VALOR } \\
\text { INSTRUMENTAL }\end{array}$ & $\begin{array}{l}\text { Até } \\
\text { anos }\end{array}$ & $\begin{array}{l}\text { Até } \\
\text { anos }\end{array}$ & \\
\hline Ambicioso & 29 & 0 & 29 \\
\hline Amoroso & 15 & 1 & 16 \\
\hline Capaz & 12 & 2 & 14 \\
\hline Honesto & 42 & 4 & 46 \\
\hline Responsável & 25 & 2 & 27 \\
\hline Outros & 90 & 5 & 95 \\
\hline TOTAL & 213 & 14 & 227 \\
\hline
\end{tabular}

Nota. Qui-quadrado=3,215; DF=5; P_valor=0,519.
Tabela 5: Análise de contingência entre o valor instrumental e o gênero

\begin{tabular}{|l|l|l|l|}
\hline & \multicolumn{2}{|c|}{ GÊNERO } & \multirow{2}{*}{ TOTAL } \\
\cline { 1 - 3 } $\begin{array}{c}\text { VALOR INSTRU- } \\
\text { MENTAL }\end{array}$ & Masculino & Feminino & \\
\cline { 1 - 3 } Ambicioso & 19 & 5 & 24 \\
\hline Amoroso & 8 & 4 & 12 \\
\hline Capaz & 12 & 1 & 13 \\
\hline Honesto & 29 & 11 & 40 \\
\hline Responsável & 11 & 11 & 22 \\
\hline Outros & 58 & 23 & 81 \\
\hline TOTAL & 137 & 55 & 192 \\
\hline
\end{tabular}

Nota. Qui-quadrado=8,574; DF=5; P_valor=0,127.

Tabela 6: Análise de contingência entre o valor instrumental e a formação anterior

\begin{tabular}{|l|l|l|l|l|}
\hline & \multicolumn{3}{|c|}{ FORMAÇÃO ANTERIOR } & \multirow{2}{*}{ TOTAL } \\
\cline { 1 - 4 } \multicolumn{1}{|c|}{ VALOR TERMINAL } & $2^{\circ}$ grau Completo & Curso Técnico Completo & Graduação Completa & \\
\hline Ambicioso & 19 & 7 & 3 & 29 \\
\hline Amoroso & 13 & 3 & 0 & 16 \\
\hline Capaz & 7 & 3 & 4 & 14 \\
\hline Honesto & 29 & 14 & 3 & 46 \\
\hline Responsável & 18 & 8 & 1 & 27 \\
\hline Outros & 71 & 20 & 4 & 95 \\
\hline TOTAL & 157 & 55 & 15 & 227 \\
\hline
\end{tabular}

Nota. Qui-quadrado=16,595; DF=10; P_valor=0,084.

\section{COMPARAÇÃO DOS RESULTADOS OBTIDOS COM ESTUDOS AN-}

\section{TERIORES}

Abrahim (2008) realizou uma pesquisa exploratória similar a esta, utilizando-se de metodologia de pesquisa similar, e através dela foi possível mapear os principais valores de estudantes dos cursos de administração e contabilidade do Centro Universitário do Pará (Cesupa) e da Pontifícia Universidade 
Católica de São Paulo (PUC-SP). Foram coletados dados de uma amostra composta por 43 discentes, na faixa dos 21 anos. Os principais resultados encontrados pela autora serão expostos a seguir para efeito de comparação. A tabulação dos dados da
RSV permitiu a autora organizar os principais valores conforme a Tabela 7. 0 ranking foi obtido através da frequência absoluta, ou seja, contabilizando quantas pessoas o classificaram como sendo o valor mais importante.

Tabela 7: Frequência absoluta, percentual válido e ranking dos valores terminais e instrumentais (mais importantes)

\begin{tabular}{|l|l|l|l|}
\hline \multicolumn{1}{|c|}{ Valores Terminais } & \multicolumn{1}{c|}{ Frequência } & \multicolumn{1}{c|}{ \% } & Ranking \\
\hline Felicidade & 7 & 16,28 & 1 \\
\hline Segurança familiar & 7 & 16,28 & 1 \\
\hline Sabedoria & 5 & 11,63 & 3 \\
\hline Liberdade & 4 & 9,30 & 4 \\
\hline Vida confortável & 4 & 9,30 & 4 \\
\hline Salvação & 4 & 9,30 & 4 \\
\hline Harmonia interior & 3 & 6,98 & 7 \\
\hline Sentimento de realização & 2 & 4,65 & 8 \\
\hline Igualdade & 1 & 2,33 & 9 \\
\hline Amor maduro & 1 & 2,33 & 9 \\
\hline Reconhecimento social & 1 & 2,33 & 9 \\
\hline Vida excitante & 1 & 2,33 & 9 \\
\hline Mundo de paz & 1 & 2,33 & 9 \\
\hline Segurança nacional & 1 & 2,33 & 9 \\
\hline Auto-respeito & 1 & 2,33 & 9 \\
\hline Mundo de beleza & 0 & 0,00 & 10 \\
\hline Prazer & 0 & 0,00 & 10 \\
\hline Amizade verdadeira & 0 & 0,00 & 10 \\
\hline & & \multicolumn{1000}{|c|}{} & \\
\hline
\end{tabular}




\begin{tabular}{|l|l|l|l|}
\hline \multicolumn{1}{|c|}{ Valores instrumentais } & \multicolumn{1}{c|}{ Frequência } & \multicolumn{1}{c|}{ Ranking } \\
\hline Honesto & 15 & 34,88 & 1 \\
\hline Responsável & 7 & 16,28 & 2 \\
\hline Animado & 4 & 9,30 & 3 \\
\hline Corajoso & 3 & 6,98 & 4 \\
\hline Prestativo & 3 & 6,98 & 4 \\
\hline Independente & 2 & 4,65 & 6 \\
\hline Auto-controlado & 2 & 4,65 & 6 \\
\hline Capaz & 2 & 4,65 & 6 \\
\hline Amoroso & 2 & 4,65 & 6 \\
\hline Ambicioso & 2 & 2,33 & 10 \\
\hline Imaginativo & 1 & 2,33 & 10 \\
\hline Intelectual & 1 & 2,33 & 10 \\
\hline Liberal & 1 & 0,00 & 11 \\
\hline Asseado & 0 & 0,00 & 11 \\
\hline Tolerante & 0 & 0,00 & 11 \\
\hline Lógico & 0 & 0,00 & 11 \\
\hline Obediente & 0 & 0,00 & 11 \\
\hline Polido & 0 & 0,00 & 11 \\
\hline & 0 & & $\mathbf{1 0 0 , 0 0}$ \\
\hline
\end{tabular}

Fonte: Abrahim (2008)

Dessa forma, em ordem de importância, a felicidade, a segurança familiar e a sabedoria, os valores terminais, enquanto que a honestidade, a responsabilidade e o ânimo representando os valores instrumentais (ABRAHIM, 2008).

Na pesquisa atual, através da análise dos valores terminais primários, seguindo a ordem de impor- tância, destaca-se a felicidade, a vida confortável e a segurança familiar. Para os valores instrumentais primários, seguindo a ordem de importância, destaca-se a honestidade, a ambição e a responsabilidade. Portanto, os resultados encontrados assemelham-se aos resultados obtidos por Abrahim (2008), mas levando-se em conta que a composição das amostras é diferente. 


\section{CONSIDERACÕ̃ES FINAIS}

O objetivo desta pesquisa foi analisar os valores pessoais em estudantes de engenharia da Universidade Federal do $A B C$ e compará-los as tendências da geração $Y$. Dessa forma, construiu-se um sistema conceitual de valores e geração $Y$ com a finalidade de analisar o comportamento esperado dos estudantes em vista das necessidades do mercado de trabalho.

Através das análises descritivas foi possível identificar os três valores terminais predominantes para os indivíduos. São eles: a felicidade, a vida confortável e a segurança familiar. Esses são os estados preferidos de ser ou estar. Os três valores instrumentais predominantes são: a honestidade, a ambição e a responsabilidade. Esses valores instrumentais são os princípios de vida usados para guiar as escolhas e chegar aos valores terminais. Análises inferenciais foram realizadas, comparando-se os valores terminais e instrumentais a alguns itens pessoais dos participantes, a idade, o sexo e a formação anterior. No entanto, não foram identificadas associações entre os valores (terminais e instrumentais) e o perfil do alunado. Isso se deve ao fato de existir homogeneidade parcial na amostra estudada.

\section{REFERÊNCIAS}

ABRAHIM, G. S. A Influência dos Valores Pessoais na Determinação das Âncoras de Carreira. 2008. Tese de Mestrado, Faculdade de Economia, Administração, Contabilidade e Atuária, Pontifícia Universidade Católica de São Paulo, São Paulo. Disponível: <http:// www.dominiopublico.gov.br/pesquisa/DetalheObraForm.do?select_action=\&co_obra=121096 $>$. Acessado em: 4 dez. 2012.
Ao comparar os resultados dessa pesquisa com outras pesquisas já realizadas, a análise de correspondência mostrou semelhanças nos resultados, podendo-se afirmar que houve semelhanças nos graus de importância atribuídos aos valores, tanto nos terminais como nos instrumentais. Este fato pode estar relacionado às tendências da geração $\mathrm{Y}$, pois as características principais dessa geração, como a honestidade, a independência e o empreendedorismo, mostram-se condizentes com os resultados obtidos, e em ambas as pesquisas o público alvo era pertencente a essa geração.

Os resultados obtidos demostram que os jovens apresentam valores que condizem com as necessidades atuais das corporações, por profissionais bem preparados, que valorizam a educação e mostram-se ambiciosos, mas mantendo-se também o foco na responsabilidade, incluindo-se a ética e os cuidados com o meio ambiente, no entanto, novas relações podem ser feitas para atribuir a influência dos valores à inclinação profissional.

CARNEIRO, N. A. Turismo de Negócios e a Geração Y no Cenário de Eventos Empresariais. 2010. Tese de Mestrado, Universidade Anhembi Morumbi, São Paulo. Disponível: <http://portal.anhembi.br/publique/ cgi/cgilua.exe/sys/start.htm?infoid=6696\&sid=140>. Acesso em: 04 dez. 2012.

DOLAN; S., \& GARCIA; S. Gestão por Valores. Rio de Janeiro: Qualitymark. 2006 
FIELDS, B., WILDER, S.; BUNCH, J., \& NEWBOLD, R. Millennial Leaders. Illinois: Buffalo Grove. 2008

GASTALDELLO, A. T. A Influência de Valores Pessoais de Executivos Brasileiros e Argentinos em Negociações Comerciais. Dissertação de Mestrado, Programa de Pós-Graduação em Administração da Universidade do Rio Grande do Sul, Porto Alegre. 1999.

GOUVEIA, V. V. et al. A Estrutura e o Conteúdo Universais dos Valores Humanos: análise fatorial confirmatória da tipologia de Schwartz. In: Revista Estudos de Psicologia. n. 6, v. 2. 2001.

HOFSTEDE, G. Culture and Organizations: software of the mind. New York: McGraw-Hill. 1997

KLUCKHOHN, C. Los valores y las orientaciones de valor em la teoria de la acción. In: Parsons, T., \& Shils, E. A. (Orgs.). Hacia una teoría general de la acción. Buenos Aires: Editorial Kapelusz, p. 435485, 1968.

KUPPERSCHMIDT, B. R. Multigeneration Employees: strategies for effective management. The Health Care Manager, 19(1), p. 65-76. 2000.

MARTIN, Carolyn A. From high maintenance to high productivity: what managers need to know about Generation Y. In: Industrial and commercial training, v. 37 (1). 2005.

MCCRINDLE, M. Understanding Generation Y. Australia: The Australian Leadership Foundation. 2002.

MINARELLI, J. A. Empregabilidade: 0 caminho das pedras. São Paulo: Gente. 1995.

PARTRIDGE, H., \& HALLAM, G. Educating the Millennial Generation for evidence based information practice. Library Hi Tech, v. 24 n. 3, p. 400-419. 2006.

PETERS, T. Reimagine! Excelência nos negócios numa era de desordem. São Paulo: Futura. 2004.
QUIVY, R; \& CAMPENHOUDT, L. V. Manual de Investigação em Ciências Sociais. 2. ed. Lisboa: Gradiva. 1998.

ROKEACH, M. Attitude Change and Behavioral Change. The Public Opinion Quarterly, vol. 30, n.4, p. 529550, 1966.

ROKEACH, M. The nature of human values. New York: Free Press. 1973

ROKEACH, M. Crenças, Atitudes e Valores: uma teoria de organização e mudança. Rio de Janeiro: Interciência. 1981.

RUGIMBANA, R. Generation Y: How cultural values can be used to predict their choice of electronic financial services. Journal of Financial Services Marketing, v. 11, n. 4, p. 301-313(13), 2007.

SCHEIN, E. H. Career Anchors: discovering your real values. San Diego: Pfeiffer \& Company. 1993.

SCHWARTZ, B; \& LACEY, H. A Formação e a Transformação de Valores. In: Revista da Faculdade de Psicologia da PUC-SP. n. 1, p. 91 - 123, 1995.

SCHWARTZ, S. H.; BARDI, A. Value hierarchies across cultures: Taking a similarities perspective. In: Journal of Cross-Cultural Psychology, 32 (2), p. 268290, 2001.

SCHWARTZ, S. H. Valores Humanos Básicos: seu contexto e estrutura intercultural. In: Tamayo, A. Porto, J. B. (Orgs). Valores e Comportamento nas Organizações. Petrópolis: Vozes. 2005.

SCHWARTZ, S. H. Value orientations: Measurement, antecedents and consequences across nations. In: Jowell, R., Roberts C., Fitzgerald, R., \& Eva G. (Eds.), Measuring attitudes cross-nationally - lessons from the European Social Survey. London: Sage. 2006. 
SHIH, W., \& ALLEN, M. Working with generation-D: adopting and adapting to cultural learning and change. Library Management, v. 28 (1/2), p. 89-100, 2007.

STACKMAN, R. W, PINDER, C. C., \& CONNOR, P. E. Values Lost: redirecting research on values in the workplace. In: Ashkanasy, N. M; Wilderom, C.P.M; \& Peterson, M.F. Handbook of Organizational Culture and Climate. USA: Sage. 2004.
STRAUSS, W., \& HOWE, N. Generations: The History of America's Future, 1584 to 2069. New York: Harper Perennial. 1992.

VERGARA, S. C. Projetos e Relatórios de Pesquisa em Administração. 9. ed. São Paulo: Atlas. 2007. 